nephron

Practice
Nephron 2017;137:302-305

DOI: $10.1159 / 000478055$
Received: April 3, 2017

Accepted after revision: June 7, 2017 Published online: June 21, 2017

\title{
Standardized Renal Endpoints for Perioperative Clinical Trials: The Standardized Endpoints in Perioperative Medicine Initiative
}

\author{
David R. Mcllroy ${ }^{a}$ Andrew D. Shaw ${ }^{b}$ Paul S. Myles ${ }^{a}$ \\ a Department of Anaesthesia and Perioperative Medicine, Alfred Hospital and Monash University, Melbourne, VIC, \\ Australia; ${ }^{b}$ Department of Anesthesiology, Vanderbilt University Medical Center, Nashville, TN, USA
}

\section{Keywords}

Acute renal injury · Kidney injury · Outcome

\begin{abstract}
Perioperative acute kidney injury is a common problem. While clinical trials seek to evaluate the impact of interventions on a variety of primary and secondary endpoints with the aim of implementing this knowledge to improve perioperative outcomes, the use of valid and relevant endpoints within clinical trials is of critical importance to achieving this goal. Suitable endpoints must be validated for the study population and in light of the clinical context under investigation while also considering regulatory requirements that govern the licensing of new therapeutic agents as well as the values of patients whose outcomes we seek to improve. Heterogeneity in perioperative clinical trial endpoints and their definitions limits the ability to compare and interpret differences in outcomes across studies or to pool outcomes from different studies in meta-analysis. The Standardized Endpoints in Perioperative Medicine (StEP) initiative is an international collaboration whose goal is to identify and recommend a suite of clearly and precisely defined endpoints across multiple domains, specifically suited for use in perioperative clinical trials. The current review describes the rationale, goals and the planned pathway of the StEP renal subgroup. Development of a set of standardized and core renal endpoints, valid and relevant for use in the perioperative
\end{abstract}

\section{KARGER}

(c) 2017 S. Karger AG, Basel

E-Mail karger@karger.com

www.karger.com/nef context, precisely defined and yet with sufficient flexibility to encourage broad uptake and application should facilitate high-quality and practice-changing perioperative research into the future.

(c) 2017 S. Karger AG, Basel

\section{Introduction}

Perioperative acute kidney injury (AKI) is a common problem with an associated increase in risk for shortterm mortality, long-term mortality and chronic kidney disease $[1,2]$. Typically occurring in $20-30 \%$ of patients undergoing cardiac surgery [3], recent data suggests that it may also occur in more than $30 \%$ of patients undergoing major non-cardiac surgeries [4]. Clinical trials seek to evaluate the impact of interventions on a variety of primary and secondary endpoints with the aim of implementing this knowledge to improve perioperative outcomes. However, the use of valid and relevant endpoints within such trials is pivotal to achieving this goal $[5,6]$.

Contribution from the AKI and CRRT 2017 Symposium at the 22nd International Conference on Advances in Critical Care Nephrology, Manchester Grand Hyatt, San Diego, Calif., USA, March 7-10, 2017. This symposium was supported in part by the NIDDK funded University of Alabama at Birmingham-University of California San Diego O’Brien Center for Acute Kidney Injury Research (P30 DK079337). 
Selected endpoints must be validated for the study population and clinical context under investigation [5] while also considering regulatory requirements for licensing novel therapeutic interventions as well as the values of patients whose outcomes we seek to improve $[7,8]$. Moreover, the consistent use of standardized and precisely defined endpoints is essential to facilitate the meaningful comparison of outcomes between studies as well as the pooling of results for subsequent meta-analysis [5].

This review describes a current, international collaboration whose goal is to identify and recommend a suite of clearly and precisely defined renal endpoints specifically suited for use in perioperative clinical trials [5]. The intent is to optimize the validity, reproducibility, comparability and utility of renal outcomes measured and reported in clinical trials from this evolving discipline.

\section{The Need for a Standardized and Core Set of Perioperative Renal Outcomes}

The critical importance of developing specialty-specific core outcomes sets, consistently reported by all clinical trials within a given specialty, is increasingly recognized [9] and embodied by the current Core Outcome Measures in Effectiveness Trials initiative (www.comet-initiative. org). The Core Outcome Measures in Perioperative and Anaesthetic Care group exists under this umbrella, seeking to develop a core outcome set for anesthesia and perioperative medicine. A European Society of Anesthetists/ European Society of Intensive Care Medicine joint taskforce recently made initial recommendations to identify and define a broad, multi-system raft of perioperative outcomes [6]. While recommending unmodified use of the kidney disease improving global outcomes (KDIGO) criteria for perioperative AKI, they acknowledged that these initial recommendations need to be broadened and refined, reflecting that the full significance of perioperative complications may require significantly longer than 30 or even 90 days after surgery to become fully known [10].

Enormous variability in renal endpoints and their definitions within existing clinical research studies helped provide the impetus for consensus statements that defined the concept of AKI more than a decade ago and unquestionably advanced the field. First published in 2004 as the RIFLE criteria, diagnostic criteria for AKI have now evolved into the KDIGO criteria, defining and staging AKI by either absolute or proportionate changes in serum

Standardized Renal Endpoints for

Perioperative Clinical Trials creatinine or increasing degrees of oliguria. While the need for uniform diagnostic criteria for AKI within clinical trials is widely agreed upon, the extent to which such uniformity has been achieved in perioperative trials remains unknown. Moreover, the question of whether current KDIGO consensus criteria for AKI provide the most appropriate renal endpoint for the perioperative setting, either alone or combined with other metrics, warrants careful consideration.

\section{What Perioperative Renal Outcomes should be Measured?}

Short-term creatinine-based outcomes are consistently associated with adverse perioperative outcomes but remain a biochemical surrogate with significant limitation [11]. Characteristic features of the perioperative period, including large volume fluid administration, poorly defined changes in creatinine production rate and an intense neuro-humoral stress response, may further limit the suitability of short-term creatinine-based or oliguria-based endpoints in this context. While calls for "hard" or longer-term outcomes are well reasoned, varied indications for the use of renal replacement therapy in clinical practice together with a low incidence of perioperative use, potential competing risk issues associated with early mortality and typically limited follow-up beyond hospital discharge highlight the challenge of selecting and defining optimal renal endpoints for perioperative trials.

The US Food and Drug Administration does not consider preventing an increase in serum creatinine sufficient evidence to register a putative nephroprotective agent [12]; instead it requires evidence that an agent reduces adverse clinical outcomes. A recent systematic review failing to find difference in rates of CKD or mortality $\geq 90$ days after surgery in clinical trials where the intervention demonstrated a significant impact on short-term serum creatinine levels [13], including the AKI sub-study of the CORONARY trial [14], supports such a stance. Although seldom reported in existing trials, observational data suggests the importance of perioperative renal outcomes beyond the incidence of AKI identified within 7 days after surgery $[12,15,16]$. The duration of AKI, biochemical evidence of renal recovery or outcomes at 30 , 60 , or 90 days postoperatively, where function is likely to have stabilized and total body creatinine returned to steady-state, may each represent important perioperative endpoints. 


\section{The Standardized Endpoints in Perioperative Medicine Initiative}

Standardized, validated and patient-centered outcome measures are the fundamental building blocks on which high-quality comparative effective trials must be based in order for evidence-based medicine to deliver enhanced outcomes for all stakeholders [5]. Developing a core set of standardized outcomes within any given perioperative domain would enhance the value of subsequent systematic reviews seeking to pool outcomes from multiple studies and thus provide greater precision of effect estimates or aid in the planning of large and definitive perioperative trials.

In 2015, a group of experienced perioperative clinical trialists met to establish a consensus process for standardizing endpoints in perioperative medicine [5]. This Standardized Endpoints in Perioperative Medicine (StEP) group is currently developing expert and consensus-based guidelines for a broad suite of precisely defined and standardized endpoints across multiple perioperative domains that will feed into the parallel development of core outcome sets for perioperative studies by the Core Outcome Measures in Perioperative and Anaesthetic Care initiative (online supplement Fig. 1, see www.karger.com/ doi/10.1159/000478055).

\section{StEP Renal Endpoints}

Under the guidance of the StEP steering committee, groups of 4-8 geographically diverse experts in perioperative medicine were assembled to address the issue of endpoints for specific perioperative domains. The renal endpoints group consists of 6 individuals, each with perioperative trials experience and background training in anesthesiology, critical care medicine or nephrology. The objective was to (1) identify renal endpoints currently used in perioperative clinical trials through a process of systematic review, (2) through appropriate summary of this literature and expert opinion, initiate a modified Delphi consensus process to propose metrics of renal injury and function best suited for use in perioperative medicine trials, and (3) incorporate the results of this process into the international StEP initiative.

After developing and agreeing on a protocol, an initial systematic review addressed the question: which endpoints are currently used to measure perioperative renal injury and function in clinical trials published in the anaesthetic, surgical, medical, critical care, cardiovascular and nephrology literature? Randomised controlled trials of perioperative interventions including $\geq 100$ patients for analysis, reporting renal outcomes and published after January 1, 2004 in a priori identified high-impact journals, were identified through Medline to facilitate a qualitative analysis of contemporary perioperative renal outcomes.

Preliminary review suggested large variation in reported outcomes across studies with definitions that were frequently short term as well as imprecise or ambiguous, with the overwhelming majority reporting the effect of interventions on prevention rather than treatment of renal injury. None of the included studies used the major adverse kidney endpoint (MAKE) composite recently described [17], and few reported any metrics of renal recovery. In parallel with this process, an initial long list of proposed renal endpoints for the perioperative community, comprising 10 endpoints ( 8 single and 2 composite) reflecting expert opinion and literature beyond published perioperative trials has been drafted without specific designation of endpoint suitability for trials of preventive vs. treatment interventions. A structured, modified Delphi process will ask participants to score each endpoint according to perceived importance. Participants will have the opportunity to add other endpoints or suggest modification to endpoints presented on this initial list. Through this process, a reduced number of precisely defined and standardized renal endpoints for perioperative clinical trials will be identified. Based on available data and expert opinion, each endpoint will then be rated on validity, reliability, feasibility and patient-centeredness.

Resource constraints together with varied aims and priorities associated with evolving phases of clinical trials mean that the most appropriate renal endpoint(s) may vary between study type and setting. To enhance the utility of proposed recommendations, it is further anticipated that a statement regarding context sensitivity will accompany each recommended endpoint. Nevertheless, the identification of one or more core endpoints, supported by expert opinion and recommended for reporting (where feasible) regardless of study phase or sample size, will enhance the value of subsequent pooled analyses for any given intervention. Results will be reported according to current recommendations [18, 19], with periodic updates anticipated as new evidence emerges. 


\section{Conclusions}

The StEP initiative is an ambitious undertaking that seeks to build on existing evidence to enhance the reporting of outcomes across all perioperative domains to the benefit of patients, clinicians, researchers and payers. Development of a set of standardized, core renal outcomes, valid and relevant for use in the perioperative context, precisely defined and yet with sufficient flexibility to encourage broad uptake and application should facilitate high-quality and practice-changing perioperative research into the future.

\section{Disclosure Statement}

D.R.M. and P.S.M. have no conflicts of interest to declare.

A.D.S. is a consultant to the following companies: Edwards, Astute and FAST Biomedical. These relationships are not relevant to the content of this manuscript.

\section{References}

1 Bihorac A, Yavas S, Subbiah S, Hobson CE, Schold JD, Gabrielli A, Layon AJ, Segal MS: Long-term risk of mortality and acute kidney injury during hospitalization after major surgery. Ann Surg 2009;249:851-858.

2 Coca SG, Singanamala S, Parikh CR: Chronic kidney disease after acute kidney injury: a systematic review and meta-analysis. Kidney Int 2012;81:442-448.

3 Englberger L, Suri RM, Li Z, Casey ET, Daly RC, Dearani JA, Schaff HV: Clinical accuracy of rifle and acute kidney injury network (AKIN) criteria for acute kidney injury in patients undergoing cardiac surgery. Crit Care 2011;15:R16.

4 Bihorac A, Brennan M, Ozrazgat-Baslanti T, Bozorgmehri S, Efron PA, Moore FA, Segal MS, Hobson CE: National surgical quality improvement program underestimates the risk associated with mild and moderate postoperative acute kidney injury. Crit Care Med 2013;41:2570-2583.

5 Boney O, Moonesinghe SR, Myles PS, Grocott MP: Standardizing endpoints in perioperative research. Can J Anaesth 2016;63:159-168.

6 Jammer I, Wickboldt N, Sander M, Smith A, Schultz MJ, Pelosi P, Leva B, Rhodes A, Hoeft A, Walder B, Chew MS, Pearse RM: Standards for definitions and use of outcome measures for clinical effectiveness research in perioperative medicine: European perioperative clinical outcome (EPCO) definitions: a statement from the ESA-ESICM joint taskforce on perioperative outcome measures. Eur J Anaesthesiol 2015;32:88-105.
7 Kalkman CJ, Kappen TH: Patient-centered endpoints for perioperative outcomes research. Anesthesiology 2015;122:481-483.

8 Palevsky PM, Molitoris BA, Okusa MD, Levin A, Waikar SS, Wald R, Chertow GM, Murray PT, Parikh CR, Shaw AD, Go AS, Faubel SG, Kellum JA, Chinchilli VM, Liu KD, Cheung AK, Weisbord SD, Chawla LS, Kaufman JS, Devarajan P, Toto RM, Hsu CY, Greene T, Mehta RL, Stokes JB, Thompson AM, Thompson BT, Westenfelder CS, Tumlin JA, Warnock DG, Shah SV, Xie Y, Duggan EG, Kimmel PL, Star RA: Design of clinical trials in acute kidney injury: report from an NIDDK workshop on trial methodology. Clin J Am Soc Nephrol 2012;7:844-850.

9 Koroshetz W: A core set of trial outcomes for every medical discipline? BMJ 2015;350:h85.

10 Jencks SF, Williams MV, Coleman EA: Rehospitalizations among patients in the medicare fee-for-service program. N Engl J Med 2009;360:1418-1428.

11 Waikar SS, Betensky RA, Emerson SC, Bonventre JV: Imperfect gold standards for kidney injury biomarker evaluation. J Am Soc Nephrol 2012;23:13-21.

12 Grams ME, Sang Y, Coresh J, Ballew SH, Matsushita K, Levey AS, Greene TH, Molnar MZ, Szabo Z, Kalantar-Zadeh K, Kovesdy CP: Candidate surrogate end points for ESRD after AKI. J Am Soc Nephrol 2016;27:28512859.

13 Coca SG, Zabetian A, Ferket BS, Zhou J, Testani JM, Garg AX, Parikh CR: Evaluation of short-term changes in serum creatinine level as a meaningful end point in randomized clinical trials. J Am Soc Nephrol 2016;27: 2529-2542.
14 Garg AX, Devereaux PJ, Yusuf S, Cuerden MS, Parikh CR, Coca SG, Walsh M, Novick R, Cook RJ, Jain AR, Pan X, Noiseux N, Vik K, Stolf NA, Ritchie A, Favaloro RR, Parvathaneni S, Whitlock RP, Ou Y, Lawrence M, Lamy A: Kidney function after off-pump or onpump coronary artery bypass graft surgery: a randomized clinical trial. JAMA 2014;311: 2191-2198.

15 Coca SG, King JT Jr, Rosenthal RA, Perkal MF, Parikh CR: The duration of postoperative acute kidney injury is an additional parameter predicting long-term survival in diabetic veterans. Kidney Int 2010;78:926-933.

16 Mehta RH, Honeycutt E, Patel UD, Lopes RD, Shaw LK, Glower DD, Harrington RA, Califf RM, Sketch MH Jr: Impact of recovery of renal function on long-term mortality after coronary artery bypass grafting. Am J Cardiol 2010;106:1728-1734.

17 Billings FT, Shaw AD: Clinical trial endpoints in acute kidney injury. Nephron Clin Pract 2014;127:89-93.

18 Diamond IR, Grant RC, Feldman BM, Pencharz PB, Ling SC, Moore AM, Wales PW: Defining consensus: a systematic review recommends methodologic criteria for reporting of delphi studies. J Clin Epidemiol 2014;67:401-409.

19 Sinha IP, Smyth RL, Williamson PR: Using the delphi technique to determine which outcomes to measure in clinical trials: recommendations for the future based on a systematic review of existing studies. PLoS Med 2011;8:e1000393.
Standardized Renal Endpoints for

Perioperative Clinical Trials
Nephron 2017;137:302-305

DOI: $10.1159 / 000478055$ 